\title{
Extracto de Compost como Control del Mildiu Lanoso (Peronospora belbahrii) y Suplemento Nutricional en Albahaca Dulce (Ocimum basilicum)
}

\author{
Olivier Francescangeli Moscoso', Alejandra Sierra Agustinus² y Dennis Roberto Ramírez \\ Castro $^{3}$
}

Resumen: Existe una fuerte tendencia hacia las alternativas agroecológicas de los fertilizantes y plaguicidas sintéticos debido al impacto que tienen en el medio ambiente y en la sociedad. El té de compost ha probado ser muy efectivo para controlar algunos patógenos. El objetivo de este estudio fue evaluar su efectividad para suprimir la incidencia de Peronospora belbahrii, un oomiceto causante de la principal enfermedad en albahaca orgánica en Zamorano, Honduras. Se fabricó una máquina para elaborar el té y se evaluaron aplicaciones foliares de este y foliares y al suelo junto con Bacillus subtilis, ácido salićlico y ácido peracético en un experimento de ocho semanas. Se inocularon las plantas con el patógeno, se aplicaron los tratamientos y se midió la incidencia semanal de la enfermedad y el rendimiento en las semanas 4 y 8 . El té de compost aplicado foliar difirió en una de los ocho semanas con el testigo, el té aplicado foliar y al suelo fue significativo en dos de las ocho semanas con el testigo y el resto de tratamientos no fueron significativos en ninguna semana. En rendimiento, el té de compost foliar + al suelo fue significativamente mayor (48.2 $\pm 6.2 \mathrm{~g} / \mathrm{planta})$ en las primeras cuatro semanas y mayor en la semana 8 , aunque sin diferencias estadísticas. Se atribuye la falta de efectividad del té de compost a los niveles altos de aditivos que causaron excesos de microorganismos lo que causó condiciones anaeróbicas que afectaron la calidad final y su habilidad supresora.

Palabras clave: Agricultura orgánica, control biológico, fertilizante, microorganismos, oomiceto.

\section{Compost Tea as a Disease Control for Downy Mildew (Peronospora belbahrii) and Nutritional Supplement in Sweet Basil (Ocimum basilicum)}

\begin{abstract}
Growing concern on the use of inorganic fertilizers and pesticides has pushed investigators to find agro-ecological alternatives due to their impact on our environment and agriculture overall. Compost tea is a tool that has proven to be effective in controlling different plant pathogens. The aim of this study was to evaluate its effectiveness in suppressing Peronospora belbahrii, an oomycete responsible for the most common disease on organic basil produced in Zamorano, where no alternatives to fungicides have been found. A compost tea brewer was built. Foliar and foliar + soil applications were evaluated as well as Bacillus subtilis, salicylic acid and peracetic acid in a continuous experiment of eight weeks. Plants were inoculated with the pathogen; treatment applications as well as disease severity evaluations were made weekly and yield was recorded at week 4 and 8 . Foliar spraying of compost tea reduced disease severity significantly when compared to the no application control in only one of the eight weeks. Foliar + soil spraying did so in only two of the eight weeks. The other treatments were not different from the control. Foliar + soil spraying of compost tea had significantly higher yields at week 4 with $48.23 \pm 6.2 \mathrm{~g} /$ plant and higher at week 8 , but not significant. The reduced affectivity of the compost tea was attributed, in part, to an elevated level of nutrients during the brewing process allowing high microorganism growth that resulted in fast oxygen consumption which could account for the lower compost tea quality.
\end{abstract}

Key words: Biological control, fertilizer, microorganisms, oomycete, organic agriculture.

1 Estudiante de maestría, Wageningen University, Wageningen, Holanda. Correo electrónico olivier.francescangeli@wur.nl

2 Instructora de Agricultura Orgánica, Escuela Agrícola Panamericana, Apartado Postal No. 93, Zamorano, Honduras. Correo electrónico asierra@zamorano.edu

${ }^{3}$ Profesor de Horticultura, Escuela Agrícola Panamericana, Apartado Postal No. 93, Zamorano, Honduras. Correo electrónico dramirez@zamorano.edu

DOI: $10.5377 /$ ceiba.v53i1.2013 


\section{Introducción}

La producción de cultivos es muy dependiente del uso de agroquímicos y fertilizantes sintéticos. Cada año, en el mundo se utilizan aproximadamente $119 \mathrm{~kg}$ de fertilizantes por hectárea cultivable y más de 2 billones de toneladas de pesticidas (FAO 2012). Esto ejerce una fuerte presión sobre el medio ambiente y está provocando cambios negativos como la degradación de los suelos y la proliferación de organismos patógenos debido a la eliminación de sus controladores biológicos naturales (Weinberg 2009).

Existen alternativas que pueden reducir la dependencia de estos compuestos sintéticos. La utilización de abonos orgánicos y extracciones líquidas es una práctica que se ha utilizado desde los años 1920 , pero que ha crecido en popularidad en los últimos años, especialmente por el desarrollo de la agricultura orgánica. Se ha demostrado que estos son capaces de suprimir enfermedades en el suelo o en la planta (Ingham 2005a, Scheuerell y Mahaffee 2002, 2004). A pesar de esto, la cantidad de estudios realizados es relativamente baja en comparación con otros tipos de control biológico (Evans et al. 2010).

El té de compost es una extracción líquida de compost de la cual se extraen los microorganismos y los nutrientes disponibles de forma aeróbica 0 anaeróbica. La forma aeróbica permite extraer y multiplicar un conjunto de microorganismos más diverso (Ingham 2005b). A pesar de haber diferentes metodologías para la preparación del té de compost aeróbico (Scheuerell y Mahaffee 2002), el principio común es poner el compost en agua con una fuente de oxigenación durante unas 24 horas con aditivos para aumentar la multiplicación de los microorganismos. Los aditivos pueden ser carbohidratos que favorecen la reproducción de bacterias, proteína que favorecen la multiplicación de hongos o una combinación de ambos.

Además de aplicarse a las hojas, el té se puede aplicar al suelo trayendo múltiples beneficios ya que mejora su actividad biológica, aporta nutrientes y crea una barrera biológica alrededor de la raíz (Ingham 2005b). Generalmente, las plantas herbáceas anuales aprovechan mejor un té con dominancia bacterial, y las plantas perennes leñosas prefieren un té con dominancia fungal (Lowenfels y Lewis 2006). El control de patógenos se logra por diversos motivos. Al haber una carga microbiana tan alta, las hojas y tallos se cubren completamente con los microrganismos por lo cual es muy difícil para un patógeno establecerse. Además, existe una alta competencia por nutrientes, parasitación directa y emisiones de metabolitos secundarios (Scheuerell 2003).

La albahaca (Ocimum basilicum L.), familia Lamiaceae, es originaria del sudeste de Asia, puede ser anual o perenne, dependiendo de la variedad. Tiene una alta importancia ya que es una de las hierbas aromáticas más utilizadas en el arte culinario. Se utiliza mucho en la cocina mediterránea debido a su fuerte sabor y alto contenido de aceites esenciales. Adicionalmente, se ha encontrado que posee propiedades antisépticas y antiinflamatorias, además de una longevidad de hasta 10 meses si se almacena bajo condiciones ideales (Sanchez Govin et al. 2000).

El hongo Peronospora belbahrii (Thines) es de la familia Peronosporaceae. El género Peronospora tiene una gran variedad de especies y muchas causan enfermedades en varias especies de plantas. Un ejemplo de esto es Peronospora destructor, uno de los principales patógenos del género Allium como la cebolla y el ajo (Rueda y Shelton 1996). A pesar de ser muy parecidos con los hongos por la producción de hifas y esporangios, los oomicetos difieren de estos en que las paredes celulares no contienen quitina, y en que son diploides (los hongos son haploides) (Heffer Link et al. 2002). Sin embargo, se descubrió que el modo en el que atacan las plantas es similar (Latijnhouwers et al. 2003), motivo por el cual se suelen llamar hongos a los oomicetos.

Peronospora belbahrii requiere alta humedad relativa ( $85 \%$ o más), temperatura moderada ( 10 a 25 ${ }^{\circ} \mathrm{C}$ ) y lluvias prolongadas para esporular (Beckerman 2009). Su ciclo de vida es de 11 a 15 días y la diseminación de esporas por el viento hace que su capacidad de extenderse sea rápida. El oomiceto se establece generalmente en la parte inferior de la hoja y provoca lesiones que pueden ser muy severas y eventualmente matar a la planta. El síntoma más visible son áreas cloróticas de color verde-amarillento en las hojas que a veces se pueden confundir con deficiencias nutricionales. El envés de la hoja se cubre de esporas de color gris a violeta y las lesiones pueden convertirse en tejido necrótico con la posterior muerte de la hoja (McGrath 2013). Todo este ciclo resulta en una reducción de los rendimientos y puede ser muy dañino en la albahaca ya que la producción de este cultivo es la producción de hojas.

Peronospora belbahrii apareció por primera vez 
en 1933 en la albahaca en Uganda. El oomiceto se contuvo durante bastante tiempo ahí, hasta que en los años 2000 hubo brotes en Suiza, Bélgica, Israel, Nueva Zelanda, Camerún y Sudáfrica. En Estados Unidos, su primera aparición fue en el sur de Florida en 2007 y desde entonces se ha expandido a la mayoría de los estados, incluyendo Hawái (Zhang et al. 2009). A pesar de que la espora se disemina con bastante facilidad con el viento, las mayores fuentes de contaminación son las exportaciones de hojas de albahaca contaminadas, al igual que la venta de semillas infestadas. Estados Unidos importa el $20 \%$ de su consumo y se demostró que el patógeno presente en este país es 100\% genéticamente igual al encontrado en Suiza (McGrath 2013).

Se ha demostrado la efectividad del té de compost para suprimir enfermedades. Scheuerell y Mahaffee (2004) evaluaron los efectos de aireación, uso de aditivos y la composición del compost sobre la eficacidad del té para controlar Pythium ultimum causante de la podredumbre de raíces en pepino. Concluyeron que todas las combinaciones reducían significativamente la enfermedad, pero que la combinación de aditivos era más determinante que la composición del compost.

El efecto de la aireación en la incidencia de Botrytis cinerea en fresas fue investigado por Welke (2004) y encontró que el té aeróbico y anaeróbico suprimían la enfermedad, pero el aeróbico generaba más rendimientos. En Australia se probó que el uso de té de compost, a partir de compost inmaduro, es efectivo para limitar la colonización de este hongo causante de la enfermedad del moho gris en frijol (Evans et al. 2010). El uso de cinco fuentes de compost extraídas en agua y suero de leche fue probado in vivo e in vitro para reducir la incidencia de Botrytis cinerea, Alternaria alternata y Pyrenochaeta lycopersici en tomate. Se encontró que todas las combinaciones reducian significativamente la incidencia de los tres patógenos (Pane et al. 2012). Igualmente, un estudio en dos variedades de uvas mostró una reducción en la expansión del Mildiú Polvoso (Erysiphe necator). Las plantas fueron inoculadas 7 días previos a la aplicación del té de compost y hubo incidencia menor a $1 \%$ en las tratadas con el té, comparado con 24 y $79 \%$ en las plantas testigo (Evans et al. 2012). Otro estudio sobre Mildiú Polvoso (Golovinomyces cichoracearum DC) en melón, demostró que el té de compost (usando ácidos húmicos y levadura como aditivos) retrasó el establecimiento e inhibió la enfermedad igual que fungicida comercial (Naidu et al. 2012).

Otros experimentos han encontrado que al usar té de compost no hubo inhibición de ciertas enfermedades. Un estudio comparó el uso de fungicidas sintéticos a base a cobre y productos orgánicos como el té de compost para controlar Septoria lycopersici en tomate y se demostró que los fungicidas sintéticos eran más efectivos en reducir la incidencia y hubo un incremento de $60 \%$ en la producción (Joslin et al. 2004). La continuación de este estudio demostró que el té de compost no tuvo ningún efecto en controlar este hongo (Bates 2007).

Al-Mughrabi (2006) determinó el efecto antagónico del té de compost en enfermedades foliares y del suelo en el cultivo de papa. La aplicación del té al suelo y foliar incrementó la incidencia de Phytophtora infestans y los tratamientos que involucraron el uso de té de compost presentaron Helminthosporium solani y Rhizoctonia solani. Además, ningún tratamiento controló Fusarium sp., Alternaria solani, Streptomyces scabiei ni Erwinia carotovora sub. carotovora. El uso del té de compost no fue efectivo para controlar en el campo a Alternaria tomatophila causante del Tizón Temprano, pero fue efectivo para controlarlo bajo invernadero y en el laboratorio (Kouyoumjian 2007). Este estudio refuerza claramente la variabilidad que existe al utilizar el té de compost para suprimir enfermedades.

Hay estudios sobre el posible aporte nutricional del té de compost. Una investigación en Chile, sobre el efecto de combinaciones de compost con o sin el té de compost y otros fertilizantes en raigrás perenne (Lolium perenne), demostró que el té solo no tenía efecto comparado con el testigo, pero al combinar este con fuentes de carbono (compost o estiércol bioestabilizado), hubo mayor eficiencia del uso del nitrógeno en todos los tratamientos (Hirzel et al. 2012). Un estudio similar evaluó el efecto nutricional del té de compost anaeróbico en fresas comparado con dos fuentes de compost y fertilizantes inorgánicos. En general, el aporte de macro y micronutrientes fue muy similar entre tratamientos, a pesar de haber ciertas diferencias en algunos elementos como el potasio, donde el aporte al suelo de los otros tratamientos fue mayor que con el té de compost (Hargreaves et al. 2009).

En la Escuela Agrícola Panamericana, Zamorano, Honduras, la Unidad de Agricultura 
Orgánica produce albahaca durante todo el año. Sin embargo, la producción se reduce considerablemente durante la primera parte del periodo de lluvias que va desde mayo hasta agosto. Esto se debe a la aparición de Peronospora belbahrii que causa la enfermedad Mildiú Lanoso. Si se pudiera controlar el daño de este oomiceto, la producción de albahaca sería mayor. Por esto, el objetivo del estudio fue evaluar el uso del té de compost y otros tratamientos orgánicos para suprimir o reducir la incidencia de este patógeno en el cultivo. Otro objetivo fue fabricar una máquina eficiente en la extracción del té de compost para lograr una cantidad y calidad de microorganismos óptima. Además, evaluar si la aplicación del té aumenta el rendimiento ya que al extraer los microorganismos del compost, extraemos también los nutrientes disponibles en el compost.

\section{Materiales y Métodos}

El ensayo se llevó a cabo entre julio y septiembre del 2013 en la Unidad de Agricultura Orgánica de Zamorano, Honduras. El lugar está localizado a $30 \mathrm{~km}$ al este de Tegucigalpa, está a $800 \mathrm{msnm}$, tiene una precipitación promedio anual de $1200 \mathrm{~mm} \mathrm{y}$ temperatura promedio de $25 \pm 2{ }^{\circ} \mathrm{C}$.

Fabricación de la máquina para elaborar el té de compost y principios del funcionamiento. Para la fabricación del extractor se utilizó como base el "simple airlift - Vortex" (Wilson s.f). El principio de este es tener una fuente de aireación en el fondo del recipiente para crear una columna de agua oxigenada que caiga dentro del tanque de agua, logrando así una circulación continua y uniforme. Para esto, se utilizó un bidón plástico de $18.9 \mathrm{~L}$ (cinco galones) como tanque de agua con un tubo central de policloruro de vinilo (PVC) de $10 \mathrm{~cm}$, todo se colocó sobre una estructura fabricada con tubos de PVC de $1.27 \mathrm{~cm}$ (Figura 1). El tubo central de PVC tiene hoyos de $1.27 \mathrm{~cm}$ de diámetro en la base y al final del tubo para la recirculación de agua, y un difusor de aire en la base conectado a una bomba de aire (112 Watts, $110 \mathrm{~L} / \mathrm{min}, \quad 0.035 \mathrm{Mpa}$ de presión) (ActiveAQUA, Hydrofarm Horticultural Products, Petaluma, California, Estados Unidos).

El principio de extracción de los microorganismos y nutrientes funciona porque el compost se encuentra en el tubo central dentro de una media de nylon en un contenedor plástico con orificios; cuando el agua y burbujas de aire, que salen de la base pasan con presión por este tubo, fuerzan la separación mecánica de las partículas del compost, extrayendo así los microorganismos y compuestos minerales. Al llegar arriba del tubo central, estos caen al tanque de agua, logrando una recirculación uniforme (Figura 2).
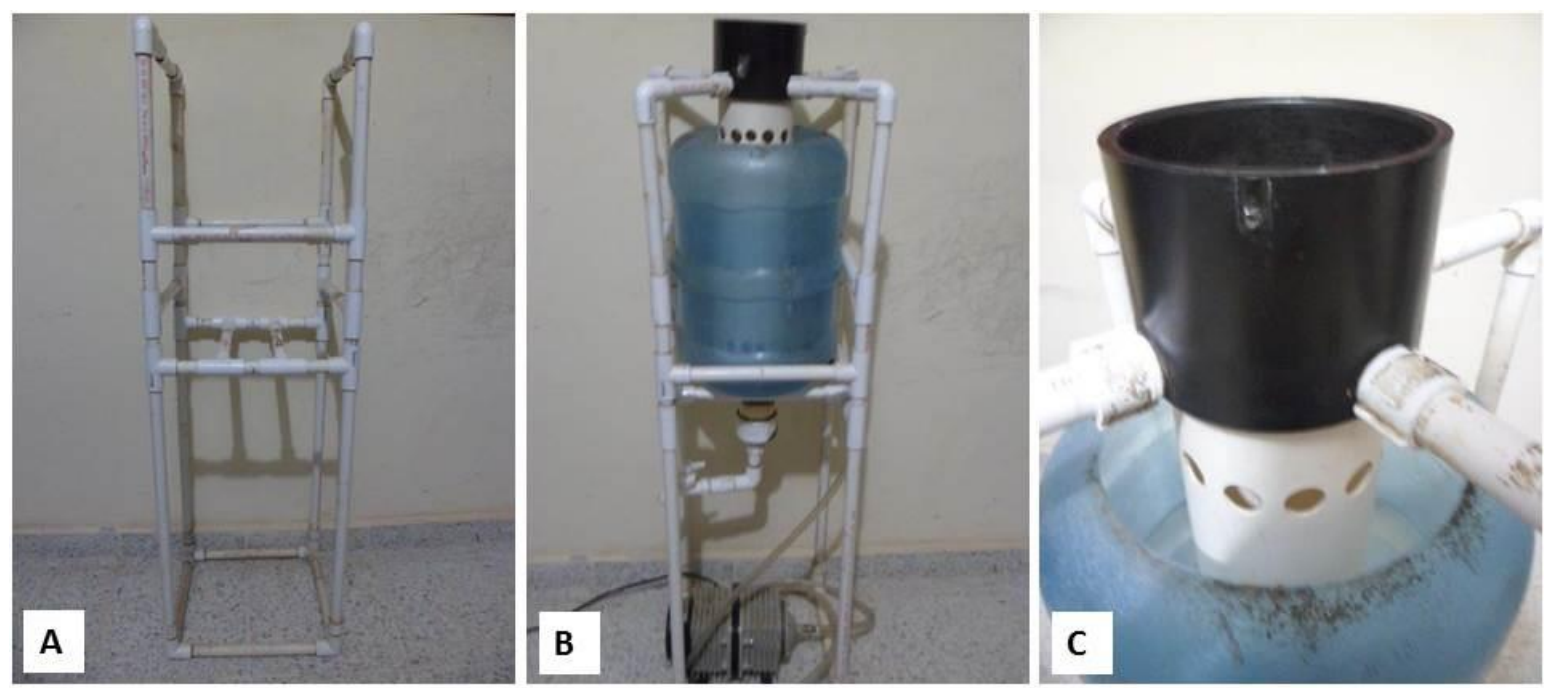

Figura 1. Máquina de extracción del té de compost: A) Estructura de PVC con tubos de 0.5 pulg; B) Bidón de agua de 5 galones en la estructura de soporte; C) Tubo central de PVC de 4 pulgadas. 

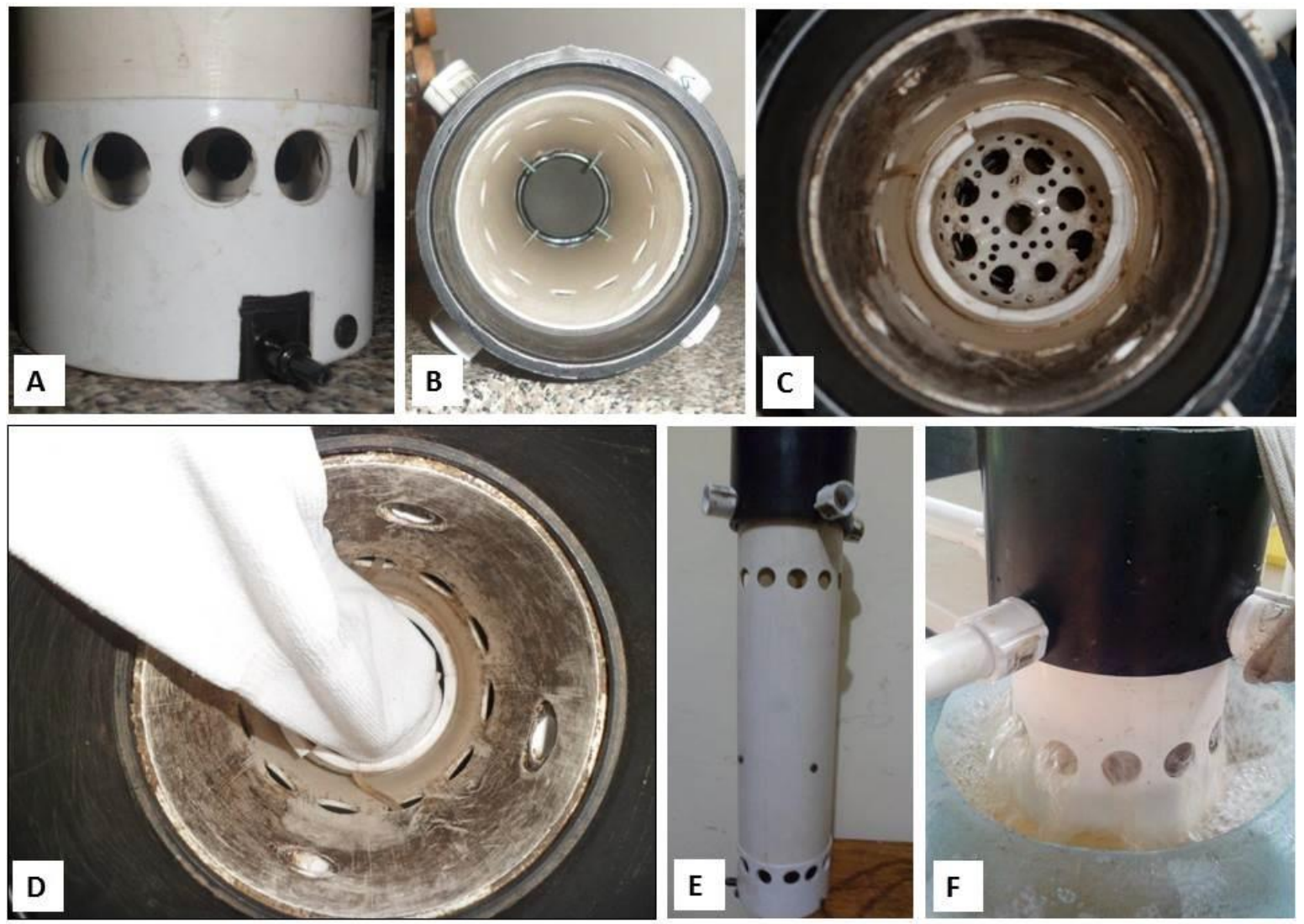

Figura 2. Funcionamiento y partes del tubo central (PVC): A) Salida difusor de aire y huecos de recirculación; B) Tubo central visto desde arriba; C) Contenedor vacío dentro del tubo central; D) Contenedor con media nylon y compost dentro del tubo central; E) Tubo central completo; F) Recirculación del té a través del tubo central.

Producción del té de compost. Se produjeron $18.9 \mathrm{~L}$ de té de compost semanalmente. Para eliminar el cloro del agua (Ingham 2005b), esta se aéreo en la máquina una hora antes de iniciar la producción del té de compost. Se determinó con un kit de medición de cloro (pH and Chlorine test kits R151083, Pentair, Los Angeles, California, Estados Unidos) que este tiempo es suficiente para eliminar los $3 \mathrm{mg} / \mathrm{L}$ de cloro que contiene el agua potable que se usó.

El requerimiento principal para la efectividad del té de compost es la reproducción eficiente de los microorganismos. La fuente de microorganismos fueron $454 \mathrm{~g}$ de una mezcla de $25 \%$ de compost, $25 \%$ de vermicompost, $25 \%$ de bocashi y $25 \%$ de olote descompuesto (todos producidos por la Unidad de Agricultura Orgánica, Zamorano, Honduras) filtrada con una media de nylon de 40 denieres.

Debido a que no existe una aparente relación entre el tipo de microorganismos y la enfermedad a suprimir (Evans et al. 2010), se estableció una relación 1:1 entre bacterias y hongos. Para esto, se añadieron $50 \mathrm{~g}$ de azúcar blanco (El Cañal, CISA, Tegucigalpa, Honduras) y $25 \mathrm{~g}$ de semolina de arroz (Planta de Concentrados, Zamorano, Honduras) al inicio de la extracción como fuentes de carbohidratos y proteínas, respectivamente (Ingham 2005b).

Se determinó que la duración ideal de esta extracción era de 72 horas ya que se obtenían más y mayor diversidad de microorganismos. Para esto, cada 12 horas se contó bajo el microscopio la cantidad de microorganismos y se determinó que hasta la hora 72 aumentaban los microorganismos, a partir de ahí las cantidades se redujeron. Se midió el oxígeno disuelto (YSI 55 Dissolved Oxygen Meter, YSI Incorporated, Yellow Springs, Ohio, Estados Unidos) (Figura 3), $\mathrm{pH}$ y la conductividad eléctrica (HI 98130 
$\mathrm{pH}$ and EC meter, Hannah Instruments, Woonsocket, Estados Unidos) (Figura 4 y 5) cada cuatro horas. El error de medición del medidor de oxígeno disuelto es de $\pm 0.3 \mathrm{mg} / \mathrm{L}$, el del $\mathrm{pH}$ es de \pm 0.02 unidades $\mathrm{y}$ el de la conductividad eléctrica es de $\pm 2.0 \%$.

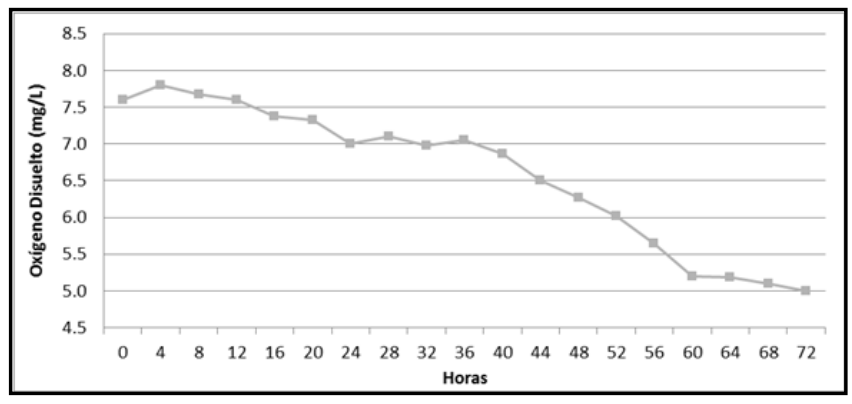

Figura 3. Niveles de oxígeno disuelto durante el ciclo de producción del té de compost.

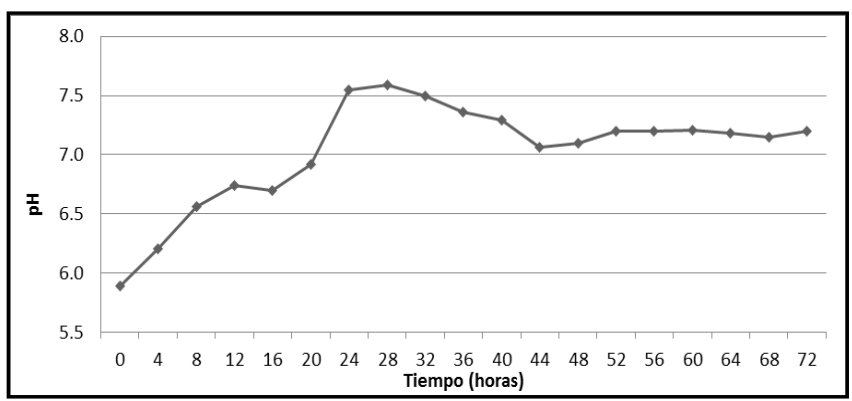

Figura 4. Variaciones en el $\mathrm{pH}$ durante el ciclo de producción del té de compost.

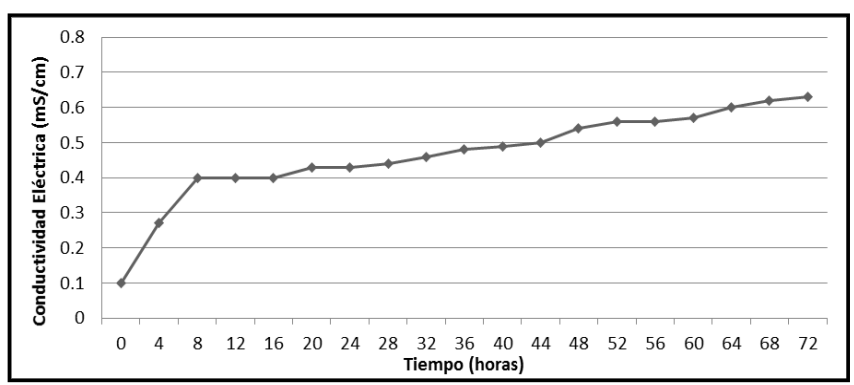

Figura 5. Cambios en la conductividad eléctrica durante el ciclo de producción del té de compost.
Debido a que la calidad del té se reduce considerablemente desde el momento que se saca de la máquina debido a la falta de oxígeno (Ingham 2005b), se aplicó inmediatamente después de sacado de la máquina. Posteriormente, se lavó la máquina solamente con agua potable para eliminar cualquier residuo ya que estos pueden crear condiciones anaeróbicas con patógenos que contaminen el siguiente ciclo de producción (Ingham 2005b).

Producción de plántulas. Se sembraron semillas de albahaca cultivar Genovesa. Se sembraron en bandejas de $62 \times 32 \mathrm{~cm}$ con capacidad de 200 celdas cada una. Se utilizó como medio de siembra Peat Moss (Pindstrup Mosebrug A/S). Se sembraron siete bandejas para garantizar una buena selección y uniformidad de plántulas al momento del trasplante. Estas plántulas pasaron 2 días en cámaras de germinación y 21 días en el invernadero.

Diseño experimental. Se utilizó un diseño de Bloques Completamente al Azar (BCA) donde se evaluaron cinco tratamientos en cuatro bloques. Se utilizaron cuatro bancales (bloques) de $15 \times 1.2 \mathrm{~m}$, orientados de norte a sur y a $1.0 \mathrm{~m}$ de distancia entre ellos. Cada unidad experimental constó de 18 plantas sembradas en bolsas plásticas negras de $22.9 \times 30.5$ $\mathrm{cm}(9 \times 12$ pulgadas $)$ espaciadas a $40 \times 40 \mathrm{~cm}$ entre ellas y a $1.0 \mathrm{~m}$ entre cada tratamiento en un bancal.

Trasplante en el campo. Durante las tres semanas previas al trasplante en el campo, se deshierbó con azadón y luego se colocó una capa de $5 \mathrm{~cm}$ de aserrín sobre el bancal. El deshierbe y colocado del aserrín se realizó una vez cada semana. Al momento del trasplante se sembró una plántula por bolsa en $800 \mathrm{~g}$ de Peat Moss (Pindstrup Mosebrug A/S) y se regó inmediatamente. Se fertilizó con $100 \mathrm{~g}$ por planta de una mezcla de $25 \%$ de compost, $25 \%$ de vermicompost, $25 \%$ de bocashi y $25 \%$ de olote descompuesto.

Inoculación. Se hicieron cuatro inoculaciones durante las dos primeras semanas después del trasplante. Se tomaron hojas de albahaca infectadas con Peronospora belbahrii y se pusieron en bolsas transparentes cerradas. Estas bolsas contenían papel toalla húmedo en el fondo y se dejaron las hojas de albahaca infectadas en la bolsa durante un día para pregerminar las esporas. Posteriormente, se lavaron las hojas con agua potable para extraer el micelio. 
Esta agua con micelio se aplicó al follaje de las plantas con una bomba de mochila (modelo P-15, Productos Tecnológicos, El Salvador) a una dosis de $10 \mathrm{ml} /$ planta. Las plantas presentaron síntomas de la infección a la tercera semana después del trasplante, momento en el cual se comenzaron a tomar datos.

Tratamientos. Hubo cinco tratamientos con una duración de ocho semanas. Sin embargo, se decidió cambiar el uso del ácido salicílico por ácido peracético en la semana cinco por lo que se dividió el ensayo en dos partes de cuatro semanas, cada uno que fue evaluado por separado (Cuadro 1).

Cuadro 1. Tratamientos evaluados durante el ensayo y dosis aplicada por semana por planta.

\begin{tabular}{ll}
\hline Tratamiento & Dosis/planta \\
\hline $\begin{array}{l}\text { Testigo (sin aplicaciones) } \\
\text { Té de compost aplicado a las }\end{array}$ & $100 \mathrm{ml}$ \\
\hline $\begin{array}{l}\text { Té de compost aplicado a las } \\
\text { hojas + al suelo }\end{array}$ & $100 \mathrm{ml}+50 \mathrm{ml}$ \\
\hline Ácido salicílico (semanas 1-4) & $25 \mathrm{mg}$ \\
\hline Ácido peracético (semanas 5-8) & $0.05 \mathrm{ml}$ \\
\hline Bacillus subtilis & $30 \mathrm{ml}$ \\
\hline
\end{tabular}

Aplicaciones. El té de compost y el ácido peracético se aplicaron con una bomba de mochila (modelo P15, Productos Tecnológicos, El Salvador) y una boquilla de abanico plano a una presión de $4 \mathrm{~kg} / \mathrm{cm}^{2}$. El té de compost fue aplicado directamente sin dilución en agua. El ácido peracético (Tsunami ${ }^{\circledR}$ 100, Ecolab Incorporated, St. Paul, Minnesota, Estados Unidos) (concentración inicial 153 ppm) fue preparado a $50 \mathrm{ppm}$ (añadiendo $0.326 \mathrm{ml}$ de Tsunami $^{\circledR} 100$ por litro de agua). Se aplicaron $50 \mathrm{ml}$ de esta dilución por planta. El ácido salićlico se diluyó a $100 \mathrm{~g}$ en $200 \mathrm{~L}$ de agua, equivalente a $25 \mathrm{mg}$ en $50 \mathrm{ml}$ de agua por planta y fue aplicado directamente a la bolsa de la planta. Todas las aplicaciones foliares tuvieron un adherente, poliéster polimetilsiloxano copolímero (Break $^{\circledR}$ Thru, BASF Mexicana, México, Distrito Federal, México) a $0.5 \mathrm{ml}$ por litro de agua. Del Bacillus subtilis (producido por Zamorano) se diluyeron $30 \mathrm{ml}$ en $70 \mathrm{ml}$ de agua y se aplicaron 100 $\mathrm{ml}$ de la mezcla en la bolsa de la planta. Todas las aplicaciones empezaron la primera semana después del trasplante.
Control de malezas, fertilización y riego. El control de malezas en las bolsas y bancales se hizo manual una vez por semana. La fertilización fue semanal aplicando $50 \mathrm{~g}$ por planta de la mezcla de $25 \%$ de compost, $25 \%$ de vermicompost, $25 \%$ de bocashi y $25 \%$ de olote descompuesto. Debido a que el estudio se realizó durante la época de lluvia, se regaron las plantas únicamente en los días que no hubo lluvia. El riego se hizo con una manguera y se regó hasta llenar completamente la bolsa con agua.

Hojas enfermas. Durante las primeras cuatro semanas, semanalmente se contaron todas las hojas enfermas por planta de 10 plantas por repetición. Se utilizó como criterio de hoja enferma únicamente la presencia de esporas en el envés de la hoja. A pesar de que esta presencia de esporas es generalmente acompañada por un amarillamiento en el haz de las hojas (McGrath 2013), esto no se tomó como criterio debido a que las deficiencias nutricionales y otras enfermedades también son causantes de un amarillamiento parcial en albahaca. Debido a que a partir de la semana cinco comenzó la segunda parte del ensayo, se decidió contar el número de hojas sanas, además del número de hojas enfermas para evaluar el porcentaje de incidencia.

Rendimiento. Se cosechó en las semanas cuatro y ocho; en la semana cuatro se utilizaron las ocho plantas restantes que no se utilizaron para contar las hojas enfermas. En la semana ocho se utilizaron estas mismas plantas, sin embargo solo se cosecharon seis plantas ya que algunas se perdieron por daño de zompopos (Atta spp.). Para la cosecha se cortaron todas las ramas a $10 \mathrm{~cm}$ desde la punta para abajo siempre y cuando tuvieran más de ese tamaño y con brotes de hojas jóvenes más abajo (Cansing Andrade y Santillán Nicola 2012).

Análisis estadístico. Los datos fueron analizados con el programa estadístico "Statistical Analysis System" (SAS. 2009) mediante un Análisis de Varianza (ANDEVA) usando el Modelo Lineal General (GLM), la prueba de separación de medias fue la de Tukey con un nivel de significancia estadística de 5\% $(P \leq 0.05)$. Dado que el análisis en el tiempo y el análisis de hojas totales presentaron una interacción significativa $(P \leq 0.05)$ entre los tratamientos con el tiempo, se ejecutaron las pruebas estadísticas separadas por semana. 


\section{Resultados y Discusión}

Hojas enfermas. Durante la primera fase del ensayo (semanas 1-4), el té de compost aplicado a las hojas tuvo menos hojas infectadas en las cuatro semanas (Cuadro 2). Sin embargo, esta diferencia no fue significativa con todos los tratamientos, en especial con el testigo que presentó medias similares durante las cuatro semanas.

La primera semana no presentó diferencias debido a que las plantas estaban pequeñas, por lo que la incidencia del oomiceto no fue muy severa para crear diferencias. Durante las semanas dos, tres y cuatro, se pudo ver un patrón en las diferencias estadísticas. Los tratamientos testigo y té de compost foliar presentaron menores tasas de infección que los tratamientos con ácido salicílico y Bacillus subtilis y el tratamiento té de compost foliar + al suelo no presenta diferencias con los otros tratamientos.

Este patrón no concuerda con los resultados de porcentaje de incidencia obtenidos en las semanas 58 (Cuadro 3). Los resultados en las semanas seis y siete mostraron diferencias significativas entre los tratamientos de té de compost y el resto. A pesar de esto, los resultados no fueron consistentes con los de la semana cinco y con los resultados de la semana ocho en donde no se encontraron diferencias entre ninguno de los tratamientos.

Al comparar la evolución entre el té de compost foliar y el testigo sin aplicaciones, se encontraron diferencias significativas solamente una de las ocho semanas (semana 7). El té de compost foliar + al suelo y el testigo sin aplicaciones solo tuvieron diferencias significativas las semanas 6 y 7 . Estos resultados son congruentes con un estudio similar que probó diferentes alternativas orgánicas (incluyendo el té de compost y Bacillus subtilis) para el control de Peronospora belbahrii en albahaca, no encontraron diferencias entre estos y el testigo sin aplicaciones (Westerveld y Filotas 2012). De la misma forma, en un estudio en papa, no se encontraron diferencias entre las aplicaciones foliares y al suelo del té de compost con el tratamiento sin aplicaciones para la supresión de hongos del suelo (Al-Mughrabi 2006). El té de compost no fue efectivo para el control de Podosphaera xanthii causante del Mildiú Polvoso en calabaza cuando fue aplicado solo, pero fue efectivo cuando se combinó con leche bovina (DeBacco 2007).

En un estudio en uvas utilizando té de compost hubo una supresión casi completa del patógeno, menos de $1 \%$ de incidencia de Erysiphe necator, comparado con $77 \%$ en el tratamiento sin aplicaciones (Evans et al. 2012). Así mismo, hubo una reducción de $75 \%$ de la incidencia de Botrytis cinerea y Alternaria alternata en tomate en comparación con el testigo (Pane et al. 2012). Estos estudios sugieren que, en caso de suprimir la enfermedad, el té de compost es efectivo y genera diferencias estadísticas notorias.

Cuadro 2. Cantidad de hojas infectadas de albahaca dulce var. Genovese por Peronospora belbahrii en las semanas 1-4.

\begin{tabular}{l|c|c|c|c}
\hline \multirow{2}{*}{ Tratamiento } & \multicolumn{4}{|c}{ Semanas } \\
\cline { 2 - 5 } & 1 & 2 & 3 & 4 \\
\hline Testigo & $3.8 \pm 4.8^{\Psi}$ & $7.5 \pm 10.0 \mathrm{~b}^{\S}$ & $34.8 \pm 13.5 \mathrm{bc}$ & $52.2 \pm 27.5 \mathrm{bc}$ \\
\hline Té de compost aplicado a las hojas & $2.6 \pm 4.8$ & $5.9 \pm 9.7 \mathrm{~b}$ & $31.3 \pm 13.6 \mathrm{c}$ & $50.2 \pm 27.2 \mathrm{c}$ \\
\hline $\begin{array}{l}\text { Té de compost aplicado a las hojas }+ \\
\text { al suelo }\end{array}$ & $4.1 \pm 4.7$ & $11.6 \pm 9.7 \mathrm{ab}$ & $41.0 \pm 13.5 \mathrm{ab}$ & $56.2 \pm 27.2 \mathrm{bc}$ \\
\hline Ácido Salicílico & & & & \\
\hline Bacillus subtilis & $5.8 \pm 4.4$ & $14.1 \pm 8.6 \mathrm{a}$ & $43.1 \pm 13.1 \mathrm{a}$ & $61.9 \pm 26.8 \mathrm{~b}$ \\
\hline \multicolumn{1}{|c|}{ Probalilidad } & $3.5 \pm 4.7$ & $10.9 \pm 9.4 \mathrm{ab}$ & $39.4 \pm 12.6 \mathrm{ab}$ & $61.9 \pm 26.8 \mathrm{~b}$ \\
\hline
\end{tabular}

${ }^{\Psi}$ Promedio y desviación estándar

$\S$ Medias con letras diferentes en la columna indican diferencias significativas $(P \leq 0.05)$ 
Cuadro 3. Porcentaje de incidencia de Peronospora belbahrii en albahaca dulce var. Genovese durante las semanas 5-8.

\begin{tabular}{l|c|c|c|c}
\hline \multirow{2}{*}{ Tratamiento } & \multicolumn{4}{|c}{ Semanas } \\
\cline { 2 - 5 } & 5 & 6 & 7 & 8 \\
\hline Testigo & $68.4 \pm 12.5^{\psi} \mathrm{ab}^{\S}$ & $39.1 \pm 11.4 \mathrm{ab}$ & $45.5 \pm 15.4 \mathrm{a}$ & $28.6 \pm 12.8 \mathrm{a}$ \\
\hline Té de compost aplicado a las hojas & $60.8 \pm 15.9 \mathrm{~b}$ & $34.6 \pm 10.2 \mathrm{bc}$ & $34.7 \pm 11.3 \mathrm{~b}$ & $32.5 \pm 17.4 \mathrm{a}$ \\
\hline $\begin{array}{l}\text { Té de compost aplicado a las hojas }+ \\
\text { al suelo }\end{array}$ & $67.2 \pm 11.9 \mathrm{ab}$ & $28.7 \pm 7.0 \mathrm{c}$ & $27.7 \pm 9.3 \mathrm{~b}$ & $27.3 \pm 12.0 \mathrm{a}$ \\
\hline Ácido Peracético & $74.0 \pm 11.2 \mathrm{a}$ & $40.9 \pm 11.9 \mathrm{a}$ & $48.7 \pm 14.0 \mathrm{a}$ & $27.1 \pm 14.3 \mathrm{a}$ \\
\hline Bacillus subtilis & $67.7 \pm 15.7 \mathrm{ab}$ & $37.9 \pm 12.0 \mathrm{ab}$ & $43.4 \pm 19.0 \mathrm{a}$ & $29.4 \pm 12.7 \mathrm{a}$ \\
\hline \multicolumn{1}{c|}{ Probalilidad } & 0.07 & $<0.001$ & $<0.001$ & 0.321 \\
\hline
\end{tabular}

${ }^{\text {P}}$ Promedio y desviación estándar

$\S$ Medias con letras diferentes en la columna indican diferencias significativas $(P \leq 0.05)$

Una de las explicaciones a estas diferencias en la efectividad del té de compost para suprimir enfermedades es que los patógenos difieren en su fisiología, biología y requerimientos para sobrevivir, por lo que responden diferente a los efectos antagónicos de otros microorganismos (Nelson y Boehm 2002; Scheuerell 2003).

Es necesario hacer conteos microbiológicos del té de compost en el laboratorio para evaluar si cumple los estándares de un té de compost capaz de suprimir enfermedades (Ingham 2005b). No se hicieron en este estudio porque no hay un laboratorio en la región capaz de hacerlos, así que la baja calidad potencial del té producido pudo ser una de las razones de su inefectividad.

Los niveles de oxígeno disuelto durante el ciclo de producción del té de compost no fueron óptimos. Niveles debajo de $6 \mathrm{mg} / \mathrm{L}$ pueden ser perjudiciales para una parte de la carga microbiana (Ingham 2005b). Este consumo creciente de oxígeno indica una proliferación y crecimiento de los microorganismos que podría considerarse positiva, sin embargo es necesario controlar la cantidad de aditivos en el té para evitar esta proliferación excesiva y las condiciones perjudiciales (Ingham 2005b). En este caso, los niveles de oxígeno disuelto bajaron hasta $5 \mathrm{mg} / \mathrm{L}$ durante las últimas 12 horas del proceso (Figura 3), lo cual sugiere que estas condiciones pueden haber sido un factor importante en reducir la efectividad del té de compost.

Hay que tomar en cuenta que la medición del oxígeno disuelto solo se realizó una vez durante todo el proceso. A pesar que se estandarizó el proceso, las condiciones ambientales y la diversidad de la fuente de microorganismos varía entre cada preparación del té de compost. Esto sugiere que los niveles bajos de oxígeno no fueron una constante, sin embargo las variaciones no pueden ser lo suficientemente grandes como para variar de manera significativa la calidad del té. Fue reportado que el $85 \%$ de la capacidad de suprimir enfermedades del té viene de la cobertura bacterial de las hojas (Ingham 2005a), pero que los niveles bajos de oxígeno afectan más a los hongos y protozoarios (Ingham 2005b). En un estudio similar, el té de compost no fue efectivo para controlar la Septoriosis en tomate (Septoria lycopersici), se atribuyó esta inefectividad, además de las variables mencionadas en este estudio, a otras variables como la temperatura, el tiempo de extracción, la hora de aplicación y la edad del compost (Bates 2007).

Hay que tomar en cuenta también el tipo de aditivos utilizados. A pesar de que las bacterias consumen azúcares simples (como el azúcar blanco utilizado en este estudio) y los hongos fuentes de proteína (como la semolina de arroz usada en este estudio) (Ingham 2005b), la mayoría de ensayos con té de compost utilizan melaza como fuente de azúcares y algas líquidas, ácidos húmicos o pescado hidrolizado como fuente de proteínas para los hongos (Bates 2007, Al-Mughrabi 2006, Scheuerell y Mahaffee 2004, Kouyoumjian 2007). No se atribuyen los aditivos usados a la baja efectividad porque cumplen con los requisitos establecidos por Ingham (2005b), pero se deben evaluar aditivos en varias concentraciones para determinar los efectos en la población microbiana.

La baja efectividad del ácido salicílico para 
controlar Peronospora belbahrii corresponde con estudios que evaluaron el uso de varios inductores de resistencia adquirida para el control de este patógeno en albahaca donde no se encontraron diferencias significativas entre el uso de ácido salicílico con el tratamiento sin ácido salicílico (Zhang et al. 2012). El uso de otro inductor de resistencia adquirida, acibenzolar-S-methyl (ASM) en dosis de $5 \mathrm{mg} / \mathrm{L}$ tres veces por semana, redujo la incidencia en $93.8 \%$ y el uso del ácido aminobutírico DL-3 en dosis de 125 $\mathrm{mg} / \mathrm{L}$ redujo la incidencia en comparación con el testigo. Al igual que en el estudio anterior, el uso de acibenzolar-S-methyl (ASM) fue efectivo para controlar la enfermedad en comparación con otros inductores de resistencia, controladores biológicos y productos naturales (Gilardi et al. 2013).

Los inductores de resistencia adquirida funcionan porque la planta al ser atacada por un patógeno externo genera proteínas y activa genes que le confieren la habilidad de memorizar la enfermedad y defenderse de esta durante unos 20 días (Conrath University 2006). Se puede constatar entonces que, en el caso del ácido salicílico en este ensayo, este mecanismo de defensa no tuvo efecto ya que la incidencia fue creciente en el tiempo (Figura 6).

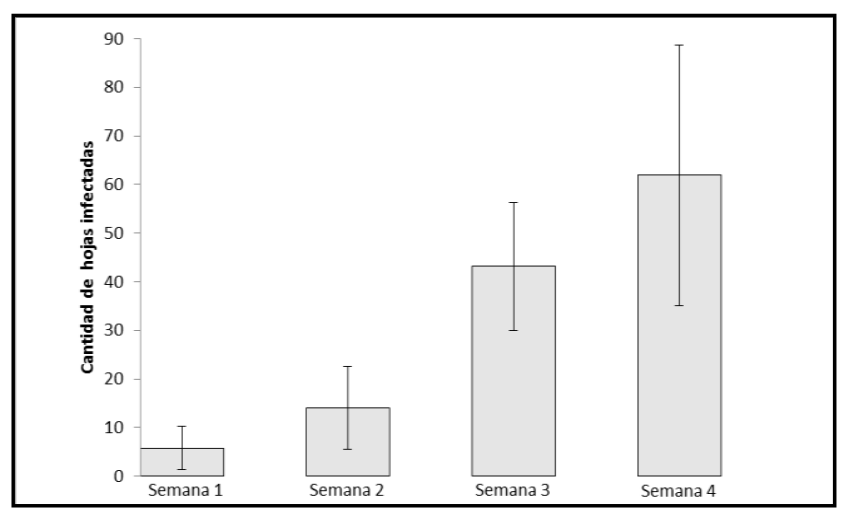

Figura 6. Cantidad de hojas de albahaca dulce var. Genovese infectadas con Peronospora belbahrii por planta en el tratamiento con ácido salicílico durante las semanas 1-4.

Al igual que los otros tratamientos, el Bacillus subtilis no controló la enfermedad. Un estudio reciente probó el uso de fungicidas a base de cobre, agentes naturales, inductores de resistencia y controladores biológicos (Gilardi et al. 2013). Al igual que en este estudio, el Bacillus subtilis no fue efectivo para controlar Peronospora belbahrii en albahaca.

En este estudio se aplicó el Bacillus subtilis directamente al sustrato, aunque se trató de controlar un patógeno que afecta la hoja. Esta bacteria protege la planta a través de la generación de biopelículas que, junto con la acción de surfactina (lipopéptido antibacterial), colonizan y protegen las raíces de la planta de los patógenos (Bais et al. 2004). Esto sugiere que su efecto funciona únicamente por contacto directo con el patógeno. Sin embargo, se ha demostrado que la presencia de la cepa FB17 de la bacteria en las raíces de Arabidopsis thaliana activa señales a través del ácido salicílico y el ácido abscísico para que la planta cierre los estomas y evite la entrada de Pseudomonas syringae (Kumar et al. 2012). Otro estudio demostró que la presencia de Bacillus spp. genera resistencia adquirida sistémica en la planta, lo cual ayuda a controlar enfermedades causadas por hongos foliares y del suelo, bacterias, virus y nematodos (Choudhary y Johri 2009).

Se ha demostrado que el uso de Bacillus subtilis es más eficiente cuando entra en contacto directo con la planta. La acción de esta bacteria para el control de mildiu lanoso y polvoso en pepino fue significativamente reducida cuando fue aplicado simultáneamente debido a la acción de metabolitos generados por el Bacillus subtilis (Elsayed et al. 2013). Otro estudio demostró que dos productos comerciales de Bacillus subtilis fueron eficaces para controlar Phytophtora infestans causante del Tizón Tardío en tomate por la acción de diferentes metabolitos, pero que este efecto se potenciaba en parte por la resistencia inducida generada por la presencia de Bacillus subtilis (Sultan 2012). Se requiere entonces, investigar más sobre el modo de acción de Bacillus subtilis en Peronospora belbahrii, para evaluar si realmente no logra controlarlo o si el problema fue el tipo de aplicación realizada.

El ácido peracético es usado en la industria como desinfectante para el agua (Flores et al. s.f), como bactericida y fungicida en el procesamiento de alimentos y se ha demostrado que es muy eficiente (Kyanko et al. 2010). Además, ha sido aprobado por la OMRI (Organic Materials Review Institute) para su uso en la agricultura orgánica. El uso del fungicida orgánico OxyDate ${ }^{\circledR}$ (peróxido de hidrógeno + ácido peroxiacético) fue recomendado para el control de Peronospora belbahrii en albahaca (McGrath 2013). Al ser aplicado libera radicales de hidroxilo y ácido 
peracético (Enviro Tech s.f) por lo que se podría predecir que este ácido puede suprimir Peronospora belbahrii. Sin embargo, no se encontraron diferencias significativas con la aplicación de este producto ni estudios que probaron el uso del ácido peracético para el control de Peronospora belbahrii. Se puede deducir entonces, que su alta tasa supresiva de patógenos de alimentos en plantas de procesamiento no se transmitió y se debe evaluar si funciona como fungicida en el campo.

Ninguno de los tratamientos fue efectivo contra Peronospora belbahrii. Debido a que es un oomiceto, no es controlado con las mismas prácticas ni fungicidas que controlan a los hongos (Westerveld y Filotas 2012). A pesar de que varios fungicidas controlan Peronospora belbahrii (McGrath 2013), se ha demostrado que el té de compost, controladores biológicos y algunos inductores de resistencia adquirida no suprimen la enfermedad significativamente (Gilardi et al. 2013, Zhang et al. 2012, Westerveld y Filotas 2012). La fácil dispersión de Peronospora belbahrii a través de zoosporas (movimiento con el agua y viento) y oosporas (pueden sobrevivir años en el tejido foliar) contribuye a la agresividad de este oomiceto y a su alta capacidad de resistencia a diferentes medidas supresivas (Sanagorski 2012). Se puede entonces, atribuir en parte la dificultad para controlar este patógeno con medidas agroecológicas a sus características genéticas y su eficiencia fisiológica.

Rendimiento. La media del tratamiento té de compost foliar y al suelo fue estadísticamente mayor al resto de tratamientos para la semana 4 (Cuadro 4). Además, al comparar los tratamientos té de compost foliar con té de compost foliar y al suelo, se obtuvo una diferencia significativa de $12.9 \mathrm{~g} /$ planta. Se puede entonces decir que, las aplicaciones al suelo de té de compost aumentan el rendimiento del cultivo.

A diferencia de los resultados anteriores, en la semana 8 no hay diferencias significativas del té de compost foliar + al suelo con el resto de los tratamientos (Cuadro 4). Sin embargo, el rendimiento de $16.3 \mathrm{~g} /$ planta del té de compost foliar + al suelo es significativamente mayor que los $7.5 \mathrm{~g} /$ planta del testigo. A pesar que los resultados en la semana 8 no muestran diferencias significativas, los resultados avalan las diferencias significativas obtenidas en la semana 4. El tratamiento té de compost foliar + al suelo tuvo más peso de hojas por planta.

En este estudio se aplicó cada semana, junto con el té de compost, una mezcla de $25 \%$ bocashi, $25 \%$ compost, $25 \%$ olote descompuesto y $25 \%$ vermicompost; los resultados son congruentes con un estudio en fresas donde se concluyó que al aplicar un fertilizante orgánico (compost 0 bioestiércol) en combinación con el té de compost, hubo mayor rendimiento que el resto de tratamientos. Los autores atribuyen el aumento en rendimientos a mayor disponibilidad del nitrógeno (Hirzel et al. 2012). Esto puede ser debido a que, al aplicar una gran cantidad de microorganismos del té de compost, la mezcla de bocashi, compost, olote y vermicompost se descompone más rápido y hay mayor disponibilidad del nitrógeno y hay mayor crecimiento.

Cuadro 4. Rendimiento promedio en gramos por planta de albahaca dulce -var. Genovese- en la semana 4 y 8.

\begin{tabular}{lccc}
\hline Tratamiento & Semana 4 & Semana 8 \\
\hline Testigo & $33.7 \pm 3.5^{\psi} \mathrm{b}^{\S}$ & $7.5 \pm 1.9 \mathrm{~b}$ \\
\hline Té de compost aplicado a las hojas & $35.3 \pm 7.5$ & $\mathrm{~b}$ & $9.2 \pm 1.4 \mathrm{ab}$ \\
\hline Té de compost aplicado a las hojas y al suelo & $48.2 \pm 6.2 \mathrm{a}$ & $16.3 \pm 6.6 \mathrm{a}$ \\
\hline Ácido salicílico & $29.5 \pm 3.7 \mathrm{~b}$ & \\
\hline Ácido peracético & & & $8.3 \pm 7.0 \mathrm{ab}$ \\
\hline Bacillus subtilis & $27.5 \pm 8.5$ & $\mathrm{~b}$ & $12.1 \pm 2.2 \mathrm{ab}$ \\
\hline \multicolumn{1}{c}{ Probabilidad } & 0.018 & 0.172 & \\
\hline
\end{tabular}

${ }^{\Psi}$ Promedio y desviación estándar.

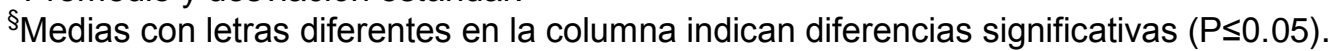


Hubo más rendimientos cuando el té de compost se aplicó al suelo y al follaje. En tomate, se aplicó té de compost solo al follaje y no hubo diferencia con el testigo sin aplicar nada (Bates 2007). Esto avala los resultados de este estudio donde no hay diferencias entre el té de compost aplicado solo a las hojas con los otros tratamientos.

El estudio de Westerveld y Filotas (2012) probó que el uso de Bacillus subtilis aumentó el rendimientos en albahaca en comparación con los otros tratamientos orgánicos, lo cual difiere del resultado encontrado en este estudio donde el Bacillus subtilis no aumentó el rendimiento comparado con los otros tratamientos. Esto se debe a que, a pesar que la bacteria puede mejorar las condiciones del suelo y aumentar la descomposición de la materia orgánica, no tiene nutrientes por lo que su aporte nutricional es mínimo y esto se traduce en menor rendimiento.

Debido a que el uso del té de compost como suplemento nutricional ha sido probado en diferentes cultivos, un estudio evaluó varias soluciones orgánicas y un testigo inorgánico en tomate sobre los rendimientos (Rangel et al. 2011). Los autores encontraron que, a pesar que el tratamiento inorgánico tuvo mayor rendimiento, el contenido de sólidos solubles en los tratamientos orgánicos fue más bajo, sugiriendo así una mejor calidad de la planta. Además, encontraron que el té de vermicompost tuvo más aporte nutricional que el té de compost. Esta puede ser una buena alternativa de investigación del uso del té como fertilizante.

\section{Literatura Citada}

Al-Mughrabi, K.I. 2006. Antibiosis Ability of Aerobic Compost Tea against Foliar and Tuber Potato Diseases. Biotechnology 5(1):69-74.

Bais, H.P., R. Fall y J.M. Vivanco. 2004. Biocontrol of Bacillus subtilis against Infection of Arabidopsis Roots by Pseudomonas syringae is Facilitated by Biofilm Formation and Surfactin Production. Plant Physiology 134(1):307-319.

Bates, M. 2007. Efficacy of Compost Tea on Septoria Leaf Spot of Tomato in Field and Greenhouse Studies. Tesis M.Sc. Horticulture. Kansas State University. Manhattan, Kansas. 36 p.

Beckerman, J. 2009. Diseases of Landscape Plants, Downy Mildew. Department of Botany and Plant Pathology, Purdue University. Consultado el 16 de mayo de 2013. Disponible en línea en http://www.extension.purdue.edu/extmedia/BP/BP-68W.pdf.
Cansing Andrade, J.F y N.A. Santillán Nicola. 2012. Producción de la albahaca dulce (Ocimum basilicum L.) utilizando cuatro densidades y dos tipos de aplicación de harina de carne como fertilizante. Proyecto especial de graduación del programa de Ingeniería Agronómica, Zamorano, Honduras. 16 p.

Choudhary, D.K. y B.N. Johri. 2009. Interactions of Bacillus spp. and plants-with special reference to Induced Systemic Resistance (ISR). Microbiological Research 164:493-513.

Conrath University. 2006. Systemic acquired resistance. Plant Signal Behavior 1(4):179-184.

DeBacco, M.J. 2007. Compost Tea and Milk to Suppress Powdery Mildew (Podosphaera xanthii) on Pumpkins and Evaluation of Horticultural Pots Made from Recyclable Fibers under Field Conditions. Tesis M.Sc. Horticulture. University of Connecticut. Stors, Connecticut. $63 \mathrm{p}$.

Elsayed, B.B., S.M.H. Kamel y M.M. Hassan. 2013. Production of Antimicrobial Metabolites by Bacillus subtilis and their Applications. Biotechnology 12:14-24.

Enviro Tech. s.f. Tech data OxyDate 2 Peroxygen Bleach. Enviro Tech Chemicals, Inc. Modesto, CA. Consultado el 18 de octubre de 2013. Disponible en línea en http://www.envirotech.com/pdf/5324\%20Tech\%20Data.p df

Evans, K.J., A.K Palmer y D.A. Metcalf. 2010. Characters of aerated compost tea from immature compost that limit colonization of bean leaflets by Botrytis cinerea. Journal of Applied Microbiology 109:34 p.

Evans, K.J., A.K Palmer y D.A. Metcalf. 2012. Effect of aerated compost tea on grapevine powdery mildew, botrytis bunch rot and microbial abundance on leaves. European Journal of Plant Pathology 135:661-673.

FAO. 2012. World Food and Agriculture. FAO Statistical Yearbook. p 26-27.

Flores, M., P. Nieres, A.E. Cassano y M.D Labas. s.f. Desinfección de agua con ácido peracético: una alternativa económica y ecológica. Instituto de Desarrollo Tecnológico para la Industria Química (INTEC). Santa Fe, Argentina. 4 p.

Gilardi, G., S. Demarchi, A. Garibaldi y M.L. Gullino. 2013 Management of downy mildew of sweet basil (Ocimum basilicum) caused by Peronospora belbahrii by means of resistance inducers, fungicides, biocontrol agents and natural products. Phytoparasitica 41(1):59-72.

Hargreaves, J.C., M.S. Adl y P.R. Warman. 2009. Are compost teas an effective nutrient amendment in the cultivation of strawberries? Soil and plant tissue effects. Journal of the Science of Food and Agriculture 89:390397.

Heffer Link, V., M.L. Powelson y K.B. Johnson. 2002. Oomycetes. The Plant Health Instructor. Consultado en línea el 10 de octubre de 2013. Disponible en https://www.apsnet.org/edcenter/intropp/LabExercises/P ages/Oomycetes.aspx 
Hirzel, J., F. Cerda, P. Millas y A. France. 2012. Compost Tea Effects on Production and Extraction of Nitrogen in Ryegrass Cultivated on Soil Amended With Commercial Compost. Compost Science and Utilization 20(2):97104.

Ingham, E.R. 2005a. Compost tea: Promises and practicalities. The IPM Practitioner: The Newsletter of Integrated Pest Management 27(9-10):1-5.

Ingham, E.R. 2005b. The Compost Tea Brewing Manual. 5 ed. Soil foodweb. Corvallis, Oregon, Estados Unidos. 79 p.

Joslin, K., H. Taber, S. Helland y M. Gleason. 2004. Control of the Foliar Disease, Septoria lycopersici, in Organic Tomato Production. HortScience 39(4):831.

Kouyoumjian, R. 2007. Comparison of Compost Tea and Biological Fungicides for control of Early Blight in Organic Heirloom Tomato Production. Tesis M.Sc. Ciencia de Plantas y Ambiente. Universidad de Clemson. Clemson, Carolina del Sur. 44 p.

Kumar, A.M., V. Lakshmanan, J.L. Caplan, D. Powell, K.J. Czymmek, D.F. Levial y H.P. Bais. 2012. Rhizobacteria Bacillus subtilis restricts foliar pathogen entry through stomata. The Plant Journal 72(4):694-706.

Kyanko, M.V., M.L. Russo, M. Fernández y G. Pose. 2010. Efectividad del Ácido Peracético sobre la reducción de la carga de Esporas de Mohos causantes de Pudrición Poscosecha de Frutas y Hortalizas. Información Tecnológica 21(4):125-130

Latijnhouwers, M., P. de Wit y F. Govers. 2003. Oomycetes and fungi: similar weaponry to attack plants. Trends in Microbiology 11(10):462-469.

Lowenfels, J. y W. Lewis. 2006. Teaming with microbes: A gardener's guide to the soil food web. Timber Press, Portland, Oregon, Estados Unidos.

McGrath, M.T. 2013. Expect and prepare for Downy Mildew in Basil. Department of Plant Pathology and PlantMicrobe Biology, Cornell University. Consultado el 16 de mayo de 2013. Disponible en línea en http://vegetablemdonline.ppath.cornell.edu/NewsArticles /Basil\%20Downy\%20Mildew-2013.pdf.

Naidu, Y., S. Meon y Y. Siddiqui. 2012. In vitro and in vivo evaluation of microbial-enriched compost tea on the development of powdery mildew on melon. BioControl 57:827-836.

Nelson, E.B. y M.J Boehm. 2002. Microbial mechanics of compost-induced disease suppression. BioCycle 43:4547.

Pane, C., G. Celano, D. Villecco y M. Zaccardelli. 2012. Control of Botrytis cinerea, Alternaria alternata and Pyrenochaeta lycopersici on tomato with whey composttea applications. Crop Protection 38:80-86.

Rangel, P.P., M.F Hernández, J.L. García-Hernández, E.R Puente, J.R.E Rivera, A.L. Herrera, M.A.S Castruita y J.O. Vidal. 2011. Evaluación de soluciones nutritivas orgánicas en la producción de tomate en invernadero. Interciencia 36(9):689-693.
Rueda, A. y A.M. Shelton. 1996. Mildiú Lanoso. Cornell University. Consultado el 16 de mayo 2013. Disponible en línea en http://web.entomology.cornell.edu/shelton/veg-insectsglobal/spanish/dmildew.html.

SAS. Statistical Analysis System. 2009. SAS User's Guide. S.A.S. version 9.1. Inst. Inc. Cary, NC.

Sanagorski, L. 2012. Downy Mildew on Impatiens. IFAS Palm Beach Extension: Environmental Horticulture. Consultado en línea el 18 de octubre de 2013. Disponible

http://palmbeachcountyextension.wordpress.com/2012/0 1/26/downy-mildew-on-impatiens/

Sanchez Govin, E., I.D. Leal López, L. Fuentes Hernández y C.A. Rodriguez Ferrada. 2000. Estudio farmacognóstico de Ocimum basilicum. Revista Cubana de Farmacia 34(3):185-197.

Scheuerell, S.J. 2003. Understanding how Compost Tea can control disease. Biocycle 44:21-25.

Scheuerell, S.J. y W.F Mahaffee. 2002. Compost tea: principals and prospects for plant disease control. Compost Science and Utilization 10(4):313-318.

Scheuerell, S.J. y W.F. Mahaffee. 2004. Compost tea as container medium drench for suppressing seedling damping-off caused by Pythium ultimum. Phytopathology 94:1156-1163.

Sultan, M. 2012. Biological control of leaf pathogens of tomato plants by Bacillus subtilis (strain FZB24): antagonistic effects and induced plant resistance. Tesis de Ph.D. Universidad de Bonn, Alemania. 144 p.

Weinberg, J. 2009. Guía para las ONG sobre los plaguicidas peligrosos y el SAICM. Consultado en línea el 10 de octubre de 2013. Disponible en http://ipen.org/sites/default/files/documents/ngo_guide_ hazpest_saicm-es.pdf

Welke, S.E. 2004. The effect of compost extract on the yield of strawberries and the severity of Botrytis cinerea. Journal of Sustainable Agriculture 25(1):57-68.

Westerveld, S. y M. Filotas. 2012. Downy Mildew of Basil Organic Management Strategies. Ontario Ministry of Agriculture and Food. Consultado el 21 de octubre de 2013. Disponible en línea http://www.omafra.gov.on.ca/english/crops/organic/news /2012/2012-03a3.htm

Wilson, T. s.f. Microbe Organics. Consultado el 20 de diciembre de 2012. Disponible en línea http://www.microbeorganics.com/\#So_You_Wanna_Buil d_A_Compost_Tea_Brewer

Zhang, S., Z. Mersha, P.D. Roberts y R. Raid. 2009. Downy Mildew of Basil in South Florida. Univer. of Florida. 2 p.

Zhang, S., Z. Mersha y R. Raid. 2012. Evaluation of systemic acquired resistance inducers for control of downy mildew on basil. Crop Protection 40:83-90.

Sometido a publicación el 6 de diciembre de 2013.

Aceptado para publicación el 19 de junio de 2015. 